\title{
Greater autism knowledge and contact with autistic people are independently associated with favourable attitudes towards autistic people
}

\author{
Alanna J. Shand, Scarlett A. D. Close and Punit Shah* \\ Department of Psychology, University of Bath, Bath, United Kingdom \\ ${ }^{*}$ Corresponding author. E-mail: p.shah@bath.ac.uk
}

(Received 30 June 2020; Revised 13 August 2020; Accepted 13 August 2020)

\begin{abstract}
Understanding individual differences in attitudes to autism is crucial for improving attitudes and reducing stigma towards autistic people, yet there is limited and inconsistent research on this topic. This is compounded by a lack of appropriate measures and multivariate analyses. Addressing these issues, using up-to-date measures and multiple linear regression, we examined the relative contributions of participant age, sex, autism knowledge, level of contact with autistic people, and autistic traits to attitudes towards autistic people. We found that greater autism knowledge and higher levels of contact, but no other variables, were uniquely predictive of attitudes towards autistic people. We conclude that, in addition to public awareness campaigns to raise knowledge of autism, it may be important to increase contact between autistic and non-autistic people to improve public attitudes towards autistic people.
\end{abstract}

Keywords: Autism; Attitudes; Autism Knowledge; Public Awareness

\section{Introduction}

There is surprisingly little research on understanding and improving attitudes to autism. Further, in the limited body of existing research, there are several issues with current understanding of the individual differences underlying attitudes towards autistic people. First, there is inconsistent evidence for and against participants' age (e.g., Kuzminski et al., 2019), sex (e.g., Cage et al., 2019), and autism knowledge and autistic traits (e.g., Mac Cárthaigh \& López, 2020) being related to their attitudes to autism. Second, there have been longstanding concerns with the measurement of autism knowledge and attitudes to autistic people (Hanel \& Shah, 2020; McClain et al., 2019). Third, there is a paucity of multivariate designs/analyses examining the unique contributions of potential factors to attitudes whilst accounting for others. For example, whilst there is relatively clear evidence for greater contact with autistic people being predictive of more favourable attitudes and prosocial behaviours towards these individuals (e.g., Payne \& Wood, 2016), this is not always accounted for when investigating other predictors of attitudes to autism.

\section{Objective}

Overall, our examination of this (limited) body of research indicates that it is currently unclear which, if any, of these factors are associated with public attitudes to autism. To help address this gap in the literature-drawing on recent advancements in measuring knowledge of and attitudes towards autism

\footnotetext{
(c) The Author(s), 2020. Published by Cambridge University Press. This is an Open Access article, distributed under the terms of the Creative Commons Attribution licence (http://creativecommons.org/licenses/by/4.0/), which permits unrestricted re-use, distribution, and reproduction in any medium, provided the original work is properly cited.
} 
(e.g., McClain et al., 2019) - we sought to investigate the relative contributions of participant age, sex, autism knowledge, level of contact, and autistic traits to attitudes towards autistic people. Identifying predictors of attitudes towards autistic people is an important step towards understanding and changing potentially negative attitudes.

\section{Methods}

Participants formed a convenience sample of 229 individuals ( $72.93 \%$ female, $25.76 \%$ male; $1.31 \%$ other) recruited using social media platforms, aged 16 to 78 years $(M=28.62, S D=15.45)$. No compensation was offered for this study, which was completed online. Participants gave informed consent and all procedures complied with the ethical standards of national and institutional committees on human experimentation and with the Helsinki Declaration of 1975, as revised in 2008. Two participants were excluded as multivariate outliers, with standardised residuals $>3 S D$ s from the mean. The pattern of results was unchanged if they were included.

After reporting their age and sex, participants completed up-to-date measures quantifying their autism knowledge (between 0-31; McClain et al., 2019), level of contact with autistic people (between 1-12; Gardiner \& Iarocci, 2014), autistic traits (between 0-10; Allison et al., 2012), and their overall attitude towards autistic people (between 0-100; Hanel \& Shah, 2020). Higher scores represented more autism knowledge, contact with autistic people, autistic traits, and favourable attitudes to autism, respectively. See Supplementary Material for more details about the measures.

\section{Results}

Inspection of the data (Supplementary Material) indicated that, despite expected deviations from a normal distribution (all Shapiro-Wilk $p<.05$ ), we had appropriate variance to explore interrelationships between variables (Table 1). Notably, autism knowledge and level of contact were not significantly correlated, yet both variables were positively associated with attitudes towards autistic people.

We had $80 \%$ power to detect small-to-medium unique associations in our regression $\left(f^{2}=.06, \alpha=.05\right)$, that is, the primary analysis. Neither multicollinearity nor heteroscedasticity was a concern. The standardised residuals broadly followed a normal distribution and were not autocorrelated (DurbinWatson statistic $=1.97, p=.81$ ). Overall, data were suitable for and submitted to multiple linear regression analysis, $F(5,220)=3.01, R^{2}=.06, p=.01$, revealing that greater autism knowledge and

Table 1. Means, Standard Deviations, and Correlations

\begin{tabular}{|c|c|c|c|c|c|c|c|}
\hline Variable & $M$ & $S D$ & 1 & 2 & 3 & 4 & 5 \\
\hline 1. Age (years) & 28.62 & 15.45 & & & & & \\
\hline $\begin{array}{l}\text { 2. Sex } \\
(0=\text { Female, } 1=\text { Male })\end{array}$ & - & - & $\begin{array}{c}.06 \\
\left(.20^{\star \star}\right)\end{array}$ & & & & \\
\hline 3. Autism Knowledge & 21.62 & 2.71 & $\begin{array}{c}-.21^{\star \star} \\
(-.10)\end{array}$ & $\begin{array}{l}-.09 \\
(-.07)\end{array}$ & & & \\
\hline 4. Level of Contact & 6.88 & 3.00 & $\begin{array}{c}.07 \\
(.09)\end{array}$ & $\begin{array}{l}-.06 \\
(-.07)\end{array}$ & $\begin{array}{r}.06 \\
(.07)\end{array}$ & & \\
\hline 5. Autistic Traits & 2.62 & 1.90 & $\begin{array}{l}-.11 \\
(-.06)\end{array}$ & $\begin{array}{r}.14^{\star} \\
\left(.14^{\star}\right)\end{array}$ & $\begin{array}{l}.01 \\
(-.001)\end{array}$ & $\begin{array}{l}.07 \\
(.07)\end{array}$ & \\
\hline 6. Autism Attitudes & 63.76 & 18.94 & $\begin{array}{c}.04 \\
(.03)\end{array}$ & $\begin{array}{l}-.09 \\
(-.06)\end{array}$ & $\begin{array}{l}.16^{\star} \\
\left(.17^{\star}\right)\end{array}$ & $\begin{array}{l}.18^{\star \star} \\
\left(.17^{\star}\right)\end{array}$ & $\begin{array}{l}-.05 \\
(-.05)\end{array}$ \\
\hline
\end{tabular}

Note. Given the distribution of our data, which deviates from a normal distribution, both Pearson's $r$ and Spearman's $r$ o (in parentheses) are reported for readers with a preference for one type of analysis over the other. ${ }^{\star} p<.05,{ }^{\star \star} p<.01,{ }^{\star \star \star} p<.001$. Three participants reporting their sex as 'other' are not included in analyses involving this variable. 
Table 2. Multiple Regression Predicting Attitudes Towards Autistic People

\begin{tabular}{lccccc}
\hline Predictor & $B[\mathrm{BCa} 95 \% \mathrm{Cl}]$ & $S E_{B}$ & $\beta$ & $t$ & $p$ \\
\hline Age (Years) & $0.07[-0.10-0.24]$ & 0.08 & 0.06 & 0.88 & .38 \\
\hline Sex (0 = Female, 1 = Male) & $-2.53[-8.06-2.92]$ & 2.87 & -0.06 & -0.88 & .38 \\
\hline Autism Knowledge & $1.10[0.15-2.06]$ & 0.47 & 0.16 & 2.34 & .02 \\
\hline Level of Contact & $1.05[0.19-1.86]$ & 0.42 & 0.17 & 2.50 & .01 \\
\hline Autistic Traits & $-0.48[-1.89-0.90]$ & 0.68 & -0.05 & -0.70 & .48 \\
\hline
\end{tabular}

higher levels of contact, but no other variables, were significant predictors of favourable attitudes towards autistic people (Table 2).

\section{Discussions}

Neither age nor sex was uniquely associated with attitudes towards autistic people. The high percentage of young female participants reduces the generalisability of this finding, but equally, it is generally consistent with recent research in larger samples (e.g., Kuzminski et al., 2019). Therefore, it is unlikely that such socio-demographic factors are particularly important predictors of attitudes to autism but could practicably be accounted for in future research. Similarly, autistic traits were not significantly associated with attitudes to autism (see also, Gardiner \& Iarocci, 2014). There are ongoing concerns with measuring autistic traits in the general population (e.g., Taylor et al., 2020), which is a potential limitation of the study and many others in our field. Nonetheless, our finding is of interest as it is often assumed that people with many autistic traits have favourable attitudes towards autistic people and would therefore be good advocates for them (e.g., Komeda, 2015). This may not be the case and warrants further investigation.

\section{Conclusions}

The main conclusion from our study is that greater autism knowledge and higher levels of contact with autistic people are independently associated with favourable attitudes towards autistic people. It is notable that effect sizes were small and the study requires replication. Nevertheless, we tentatively suggest that increasing public knowledge of autism has the potential to improve attitudes towards autistic people. Equally, we suggest that increasing contact between autistic and non-autistic people may have an independent influence on attitudes towards autistic people, potentially via a different mechanism. Better understanding of their (separable) contributions to attitudes towards autistic people will be an interesting avenue in future research.

Acknowledgements. We thank Celyn Gabe-Jones and Katarzyna Konczewska for assistance with data collection, and Lucy A. Livingston for comments on a previous version of this manuscript.

Author Contributions. PS, AS, and SC conceived the study. AS and SC collected the data. PS, AS, and SC analysed the data and wrote the article. AS and SC contributed equally to this work.

Funding Information. PS is supported by the GW4 Generator Fund.

Conflicts of Interest. All authors declare no conflicts of interest.

Data Availability Statement. Data are available as Supplementary Material.

Supplementary Materials. To view supplementary material for this article, please visit http://dx.doi.org/10.1017/exp.2020.46. 


\section{References}

Allison, C., Auyeung, B., \& Baron-Cohen, S. (2012). Toward brief“"red flags" for autism screening: The short autism spectrum quotient and the short quantitative checklist in 1,000 cases and 3,000 controls. Journal of the American Academy of Child \& Adolescent Psychiatry, 51, 202-212. https://doi.org/10.1016/j.jaac.2011.11.003.

Cage, E., Di Monaco, J., \& Newell, V. (2019). Understanding attitudes and dehumanisation towards autistic people. Autism, 23, 1373-1383. https://doi.org/10.1177\%2F1362361318811290.

Gardiner, E., \& Iarocci, G. (2014). Students with autism spectrum disorder in the university context: peer acceptance predicts intention to volunteer. Journal of Autism and Developmental Disorders, 44, 1008-1017. https://doi.org/10.1007/s10803-0131950-4.

Hanel, P. H. P., \& Shah, P. (2020). Simplifying the measurement of attitudes towards autistic people. Experimental Results, 1, 1-6. https://doi.org/10.1017/exp.2020.36.

Komeda, H. (2015). Similarity hypothesis: Understanding of others with autism spectrum disorders by individuals with autism spectrum disorders. Frontiers in Human Neuroscience, 9, 124. https://doi.org/10.3389/fnhum.2015.00124.

Kuzminski, R., Netto, J., Wilson, J., Falkmer, T., Chamberlain, A., \& Falkmer, M. (2019). Linking knowledge and attitudes: determining neurotypical knowledge about and attitudes towards autism. PloS One, 14, e0220197. https://doi.org/10.1371/ journal.pone.0220197.

Mac Cárthaigh, S., \& López, B. (2020). Factually based autism awareness campaigns may not always be effective in changing attitudes towards autism: evidence from British and South Korean nursing students. Autism, 24, 1177-1190. https://doi.org/ $10.1177 / 1362361319898362$.

McClain, M. B., Harris, B., Schwartz, S. E., Benallie, K. J., Golson, M. E., \& Benney, C. M. (2019). Development and validation of the autism spectrum knowledge scale general population version: preliminary analyses. Journal of Autism and Developmental Disorders, 49, 3007-3015. https://doi.org/10.1007/s10803-019-04019-8.

Payne, J. L., \& Wood, C. (2016). College students' perceptions of attributes associated with autism spectrum disorders. Communication Disorders Quarterly, 37, 77-87. https://doi.org/10.1177/1525740114560883.

Taylor, E. C., Livingston, L. A., Clutterbuck, R. A., \& Shah, P. (2020). Psychometric concerns with the 10-item AutismSpectrum Quotient (AQ10) as a measure of trait autism in the general population. Experimental Results, 1, e3. https://doi.org/ 10.1017/exp.2019.310.1017/exp.2019.3.

Cite this article: Shand AJ, Close SAD, Shah P (2020). Greater autism knowledge and contact with autistic people are independently associated with favourable attitudes towards autistic people Experimental Results, 1, e46, 1-8. https://doi.org/ 10.1017/exp.2020.46 


\section{Peer Reviews}

\section{Reviewing editor: Dr. Hannah Schacter}

Wayne State University, Psychology, 5057 Woodward Avenue, Detroit, Michigan, United States, 48202-3489

This article has been accepted because it is deemed to be scientifically sound, has the correct controls, has appropriate methodology and is statistically valid, and met required revisions.

doi:10.1017/exp.2020.46.pr1

\section{Review 1: Greater autism knowledge and contact with autistic people are independently associated with favourable attitudes towards autistic people}

Reviewer: Rachel Moseley

Date of review: 05 July 2020

(C) The Author(s), 2020. Published by Cambridge University Press. This is an Open Access article, distributed under the terms of the Creative Commons Attribution licence (http://creativecommons.org/licenses/by/4.0/), which permits unrestricted re-use, distribution, and reproduction in any medium, provided the original work is properly cited.

Conflict of interest statement. No conflict of interest.

Comments to the Author: Congratulations on a good and important study. Minor edits to improve:

Introduction: I realise you are limited by word count, but if you can, I recommend being more explicit about HOW variables like age and sex affect attitudes towards autistic people (i.e. attitudes more positive if a person is older or younger, male or female?); and why this is important (e.g. negative impact of stigma).

Methods: Are participants university students? Was the study completed online?

Results: Table 1 hard to see where means and SD end and correlations begin. Even a dotted line or something to separate out these two parts of the table would help.

-Please specify the type of MLR - enter method, presumably?

- Not clear why you use both Spearman's Rho and Pearson's $r$ - can you explain this?

Discussion: small thing, but the last sentence does not sit quite right with me I recommend “... assumed that people with many autistic traits have putatively favourable attitudes towards autism and would thus be good advocates for autistic people".

Conclusion: I would remove "and additive" as to me it implies a statistical change that you don't report (e.g. a change in R squared).

\section{Score Card}

\section{Presentation}


Does the abstract correctly embody the content of the article? (25\%)

Does the introduction give appropriate context? (25\%)

Is the objective of the experiment clearly defined? (25\%)

Analysis

Does the discussion adequately interpret the results presented? (40\%)

Is the conclusion consistent with the results and discussion? (40\%)

Are the limitations of the experiment as well as the contributions of the experiment clearly outlined? (20\%) 


\title{
Review 2: Greater autism knowledge and contact with autistic people are independently associated with favourable attitudes towards autistic people
}

\author{
Reviewer: Dr. Eleanor Palser (1D)
}

Date of review: 28 July 2020

(c) The Author(s), 2020. Published by Cambridge University Press. This is an Open Access article, distributed under the terms of the Creative Commons Attribution licence (http://creativecommons.org/licenses/by/4.0/), which permits unrestricted re-use, distribution, and reproduction in any medium, provided the original work is properly cited.

Conflict of interest statement. Reviewer declares none

Comments to the Author: This a well-written and concise manuscript on an important topic. The methods and analyses are valid and the conclusions well-supported. I have a few comments below.

1) I wonder if the authors could say something about the direction of the effect of contact with autistic people on attitudes in the final sentence of the introduction, i.e., does increased contact generally result in more positive attitudes?

2) I would like a bit more detail on what scores on the measures are indicative of. For example, what does a score of 1 (lowest score) or 12 (highest score) on the level of contact score represent? Is it along the lines of "I've never met an autistic person"/ "I live with an autistic person"? Similarly the attitudes scale. This is particularly important because the reference for this scale is not yet published so the reader cannot refer to another article for more information. I appreciate the word limit and suggest the authors could address this point in the Supplementary Materials.

3) Are there any reliability or validity metrics available for the measures of autism knowledge, attitude etc? Alternatively, perhaps the authors could provide an indication of this in their own sample.

4) There is inconsistency in the number of decimal places used in statistical reporting.

5) A plot of the significant associations between attitudes and contact/knowledge would be helpful.

\section{Score Card}

Presentation

Is the data presented in the most useful manner? (40\%)

Does the paper cite relevant and related articles appropriately? (30\%)

\section{Context}

Does the title suitably represent the article? (25\%)

Does the abstract correctly embody the content of the article? (25\%)

Does the introduction give appropriate context? (25\%)

Is the objective of the experiment clearly defined? (25\%) 
Are the limitations of the experiment as well as the contributions of the experiment clearly outlined? (20\%) 